\title{
Didáctica de las salidas de campo de geografía en las distintas etapas educativas: Primaria, Secundaria, Grado, Postgrado, Formación Abierta y Profesional
}

Juan Garrido Clavero - Universidad de Jaén

Miguel Ángel Sánchez del Árbol - Universidad de Granada

Gabriel Jesús Fernández Adarve - Universidad de Granada

\author{
(i) $0000-0002-8346-8228$ \\ (D) 0000-0002-6507-4030 \\ (1) 0000-0001-6379-7625
}

Recepción: 10.04.2020 | Aceptado: 30.06.2020

Correspondencia a través de ORCID: Juan Garrido Clavero

iD 0000-0002-8346-8228

Citar: Garrido-Clavero J., Sánchez-del Árbol MA., Fernández-Adarve GJ., (2020) Didáctica de las salidas de campo de geografía en las distintas etapas educativas: Primaria, Secundaria, Grado, Postgrado, Formación Abierta y Profesional. REIDOCREA, 9, 155-172.

Resumen: Introducción: una adecuada didáctica de la Geografía, así como de las demás disciplinas territoriales (urbanismo y ordenación del territorio), requiere de la realización de salidas de campo para contribuir a la comprensión y aprendizaje de las interrelaciones físicas y humanas que suceden en el territorio. Objetivos: Pero éstas exigen una preparación didáctica muy específica, pues ha de combinar múltiples factores; factores que cambian según la etapa educativa de que se trate. Metodología: se presentan algunas de las principales diferencias surgidas en la confección de los itinerarios didácticos a seguir en las salidas de campo de la Geografía impartida en la enseñanza primaria, secundaria, la docencia universitaria de grado y postgrado, la docencia universitaria no reglada y la formación científico-técnica posterior. Resultados: en el trabajo de investigación realizado se ha valorado cada una de las iniciativas planteadas en la confección de los itinerarios didácticos de las salidas de campo. Conclusiones: con la observación de estas diferencias, se advierte sobre la necesidad de adaptar a cada nivel educativo los respectivos itinerarios didácticos.

Palabra clave: Territorio

Didactic of geography field trips in the different educational stages: primary, secondary, grade, postgraduate, open and professional training.

Abstract: Introduction: an adequate teaching of Geography, as well as other territorial disciplines (urban and territorial planning), requires the realization of field trips to learn the physical and human interrelations that occur in the territory. Objectives: But these require a specific propaedeutic to combine multiple factors. However, these factors are not the same according to the educational level considered. Methodology: Thus, the following are some of the main differences to which attention must be paid when preparing the didactic itineraries of the field trips of the Geography of primary and secondary education, the undergraduate and postgraduate university teaching, the unregulated university teaching and the subsequent training acquired in the scientific-technical forums. Results: In the research work carried out, each of the initiatives proposed in the preparation of the educational itineraries of the field trips has been evaluated. Conclusions: With the observation of these differences, it is intended to warn about the need to adapt the respective educational itineraries to each educational level.

Keyword: Territory

\section{Introducción}

En Geografía, como en otras ciencias sociales y espaciales, la salida de campo es un importante recurso didáctico en los procesos de enseñanza-aprendizaje (Sala y Batalla, 1996, p.303), si bien cabe precisar que no es el único y, para determinados aspectos o conocimientos, ni siquiera resulta indispensable, como es el caso de las técnicas desarrolladas en laboratorio, los estudios fundamentados esencialmente en el empleo de TIGs o los análisis realizados bajo parámetros estrictamente cuantitativos, por citar sólo unos ejemplos (García-Ballesteros, 1986). Sin embargo, es pertinente recordar que las salidas de campo, y el correspondiente trabajo de campo que apareja, cuentan con 
una larga tradición en la disciplina geográfica, yendo desde los procesos de explo ración sistemáticos realizados a partir del siglo XIX por la Royal Geographical Society, la Société de Géographie y más tardíamente por la Real Sociedad Geográfia (emersión del positivismo científico aunado con los procesos de neo-colonización imperialista), hasta la necesidad de involucrarse directamente en las problemáticas socio-espaciales de cada momento y cada lugar, pasando por la observación directa de hechos y fenómenos territoriales sucedidos (Zusman, 2011). Pero a esos trabajos de campo con intencionalidad esencialmente investigadora y aplicada se agregan los de motivación pedagógica, por lo que, con el tiempo, las salidas y los trabajos de campo con discentes se han convertido en una alternativa educativa a la tradicional clase magistral de aula (Sousa, García-Monteagudo y Souto, 2016, p.2) o, cuando menos, en un importante complemento a la educación geográfica; reforzando de esta manera la que puede ser una de sus principales funciones de la Geografía: la de servir de puente entre las ciencias naturales y las ciencias sociales (Pulgarín, 2000, p.6). Más aún, el análisis e interpretación del paisaje y el territorio constituyen uno de los objetivos principales de la Geografía, por lo que su observación directa y el desarrollo de determinadas actividades por parte del alumnado se erigen como un proceso pedagógico por excelencia (García Ruiz, A., 2013).

Para un correcto desarrollo de estas salidas de campo es necesario que sean prediseñadas convenientemente (Sousa, García-Monteagudo y Souto, 2016, p.5), de forma que el trabajo de campo al que han de dar lugar resulte lo más provechoso posible didácticamente hablando, ya que, normalmente, no se dispondrá de la posibilidad de volver a repetir la práctica. En este sentido, su planificación es fundamental, debiendo responder a las especificidades del grupo discente en cuestión. Este grupo puede contener características tan particulares como el perfil de los asistentes (en términos de edad, de condición física, de nivel motivación personal, etc.) y las circunstancias en las que se realice la salida (temática o finalidad de la misma, tareas discentes a realizar, medio de transporte, incluido el pedestre; duración temporal, características del destino, etc.), hechos que requieren de una adaptación ex professo para cada caso y, por tanto, es difícil concretar denominadores comunes por la amplitud y heterogeneidad de la casuística. En cambio, lo que sí permitiría establecer bloques bien diferenciados de docencia es la distinta etapa educativa a la que van dirigidas las salidas de campo.

\section{Objetivos}

Con la presente propuesta se pretende contribuir a la adaptación del diseño de itinerarios didácticos de las salidas de campo de Geografía a las distintas etapas del sistema educativo español, atendiendo a las peculiaridades de cada nivel y con base en la experiencia precedente y directa de los autores. De esta forma, el objetivo principal de este trabajo consiste en proponer una batería de consideraciones básicas a tener en cuenta en las salidas de campo de la disciplina geográfica para cada etapa educativa, en el que se tengan en cuenta tanto los conocimientos a transmitir, como la mejor manera en que éstos pueden ser transmitidos, en el marco de procedimientos interactivos docentes-discentes de cada nivel educativo. Por consiguiente, no se trata de establecer una guía para la orientación de las salidas de campo al modo del célebre Cuestionario de excursiones a poblaciones de Manuel Bartolomé Cossío para la Institución Libre de Enseñanza, sino que más bien se trata de una revisión adaptativa de cómo han de realizarse estas salidas en cada etapa de la educación reglada.

Por su parte, algunos de los objetivos particulares planteados en esta investigación consisten en atender a tales propósitos en: 
- La Enseñanza Primaria, por mediación de la incorporación a las Unidades Didácticas Integradas de las competencias claves a adquirir en las salidas de campo del alumnado, las cuales forman parte de la Programación anual de cada maestro/maestra.

- La Enseñanza Secundaria Obligatoria y la Formación Profesional, a través de la formación básica de su profesorado realizada en el máster que los habilita como futuros docentes.

- La enseñanza universitaria de grado, mediante la toma en consideración de las principales conclusiones que se esgrimirán en la próxima Modificación del Verifica del Grado de Geografía y Gestión del Territorio de la Universidad de Granada.

- La docencia universitaria de postgrado, intentando incorporar ciertas pautas en las salidas de campo que alternativamente se suceden cada año con los alumnos matriculados en el "Máster Universitario en Análisis y Gestión del Territorio: Planificación, Gobernanza y Liderazgo Territorial", de las universidades de Granada y Rovira i Virgili de Tarragona.

- La docencia universitaria no reglada, añadiendo sus recomendaciones en la salida de campo prevista en la asignatura "Geografía y recursos territoriales del Sureste Ibérico" del Aula Permanente de Formación Abierta de la Universidad de Granada.

- La formación técnico-profesional posterior, aportando las principales conclusiones de este estudio al diseño de los itinerarios que se vayan a realizar cuando se prevean futuras actividades de difusión geográfica.

Por último, hay que aclarar que el presente trabajo forma parte de la investigación realizada para el I Congreso Internacional sobre Metodologías activas, modelos de enseñanza-aprendizaje e investigación en Ciencias de la Educación y del Deporte, la cual cristalizó en la presentación de una comunicación oral con el título "Itinerarios didácticos en las salidas de campo de Geografía. Didáctica activa en cinco niveles de enseñanza". Una de las principales conclusiones a las que se llegó en esta comunicación fue que sería muy recomendable ampliar el análisis a todo el ciclo formativo estudiantil en el que se imparte Geografía, incorporando la Enseñanza Primaria, que se había dejado aparte por no prestar los autores docencia en este nivel educativo. Es por ello que ahora, con la incorporación al elenco de investigadores de una especialista en Enseñanza Primaria, se aborda este trabajo de manera integral, cubriendo así la práctica totalidad de la labor formativa en la que se imparte la disciplina de la Geografía. Lo que ha permitido aprender e incorporar nuevos puntos de vista a la misma.

\section{Metodología}

El ámbito de trabajo de la presente aportación se circunscribe a las seis grandes etapas educativas de la educación andaluza en las que se suelen confeccionar itinerarios didácticos para la realización de las salidas de campo de geografía, urbanismo y ordenación del territorio, aprovechando que los autores y colaboradores imparten, en unos o en otros o en varios casos, docencia en:

- La enseñanza primaria, para lo que se ha contado con la colaboración de una docente-tutora en un Colegio de Educación Infantil y Primaria público dependiente de la Junta de Andalucía, en concreto el CEIP San Juan de Dios de Granada (2015-2020). Con una media de 25 alumnos por curso, lo que supone un total de 6 cursos y 150 alumnos aproximadamente testados.

- La enseñanza secundaria, de forma indirecta como docente en el "Máster Universitario Oficial de Profesorado de Educación Secundaria Obligatoria, 
Bachillerato, Formación Profesional y Enseñanza de Idiomas. Especialidad en Enseñanza y Aprendizaje de las Ciencias Sociales. Itinerario Geografía e Historia" de la Universidad de Granada (2010-2020). Con más de 100 alumnos por edición, lo que hace un total de 11 cursos y sobre unos 1.000 alumnos testados.

- La docencia universitaria, como profesor del "Grado de Geografía e Historia" y del "Grado de Arqueología", de la Universidad de Jaén (2019-2020). Con 64 alumnos.

- La docencia universitaria, como profesor del "Grado en Geografía y Gestión del Territorio" y del "Grado en Ciencias Ambientales" -en este segundo caso específicamente con la asignatura "Ordenación del Territorio"- de la Universidad de Granada (2010-2020). Con una media de 50 alumnos en Geografía y 80 en Ambientales por curso, lo que supone más de 1.000 alumnos considerados.

- La docencia universitaria, como profesor del "Grado de Edificación" de la Universidad de Granada (2007-2020). A razón de 40 alumnos por curso, lo que supone más de 520 alumnos de grado analizados.

- La docencia universitaria de postgrado, como profesor de TFM (intervención urbanística en el Albaicín y el Sacromonte) y tutor de prácticas de alumnos del "Máster Universitario en Rehabilitación Arquitectónica" y Máster Universitario en Tutela del Patrimonio Histórico-Artístico "El Legado de Al Ándalus" de la Universidad de Granada. (2018-2019), lo que supone 30 alumnos de posgrado analizados.

- La docencia universitaria no reglada, como docente en el Curso Superior de Especialización en Urbanismo y Desarrollo Sostenible de la UIM, Unión Iberoamericana de Municipalistas, (de 2006 a 2011) a razón de 30 alumnos por curso que suponen 150 alumnos analizados

- La docencia universitaria de postgrado, como profesor del "Máster Universitario en Análisis y Gestión del Territorio: Planificación, Gobernanza y Liderazgo Territorial" de la Universidad de Granada y la Universidad Rovira i Virgili (20152020). A razón de 20 alumnos por curso, lo que supone más de 100 alumnos de postgrado analizados.

- La docencia universitaria no reglada, como docentes en el "Aula Permanente de Formación Abierta" de la Universidad de Granada (2001-2020). Con una media por curso que supera la centena de estudiantes, es decir, más de 1.000 en total.

- Y la posterior formación investigadora, técnica y profesional adquirida en los distintos foros existentes (congresos, jornadas, mesas de trabajo, etc.), al iniciarse la carrera laboral en planificación, seguimiento y evaluación de planes y proyectos territoriales, urbanísticos, medioambientales, etc.

En consecuencia, la población de estudio trabajada ha sido los alumnos de 72 cursos (6 de Primaria, 11 de orientación a la Secundaria, 34 de Grado, 6 de Postgrado y 15 de docencia no reglada), lo que supone haber considerado la opinión de más de 4.000 estudiantes (aproximadamente 150 de Primaria, 1.000 de orientación a la Secundaria, 1.584 de Grado, 130 de Postgrado y 1.150 de docencia universitaria .no reglada).

Las fuentes e instrumentos consultados y trabajados para este artículo han sido el Informe del Programa Internacional para la Evaluación de Estudiantes para Andalucía (OCDE, 2016)- en este caso en lo concerniente al área de competencia de ciencias naturales por ser la de mayor asimilación a la disciplina geográfica de entre las elegibles-, la Modificación del Verifica del Grado de Geografía y Gestión del Territorio de la Universidad de Granada (DEVA, 2016) y los cuestionarios de satisfacción del Sistema de Evaluación de Asignaturas del Aula Permanente de Formación Abierta (Vicerrectorado de Enseñanzas de Grado y Postgrado de la Universidad de Granada, 2014-2015, 2015-2016, 2016-2017, 2017-2018 y 2018-2019), así como otras fuentes 
fundamentadas en las experiencias personales adquiridas por los autores en las asignaturas que imparten o en los trabajos aplicados en los que han participado, se ha procedido a su consideración de forma genérica para cada uno de estas etapas educativas, con independencia de que hayan tenido lugar en unos u otros. Sólo así, cruzando todas las deficiencias o insuficiencias detectadas en los distintos niveles educativos considerados, se puede prever su incidencia para cada nivel, abriéndose la posibilidad de plantearle soluciones específicas para cada caso.

Por consiguiente, se trata de una metodología que va desde la recogida de información con carácter inductivo, acerca de las citadas deficiencias o insuficiencias detectadas en relación a las salidas de campo, hasta la determinación de ciertos denominadores comunes obtenidos a partir del esfuerzo deductivo que aporta, no sólo la experiencia docente de los autores en cada etapa, sino también la participación de los mismos en distintos procesos de evaluación docente, como la del Equipo Técnico de Coordinación Pedagógica, la coordinación del Grado de Geografía y Gestión del Territorio, la pertenencia a distintos comités científicos de congresos o la participación en diferentes comisiones de evaluación de grado y postgrado. De todas estas experiencias se ha hecho una criba, primero seleccionando todo lo que guardaba relación con las salidas de campo y después vinculando cada pronunciamiento a cada una de las iniciativas consideradas, hasta valorar su repercusión para cada etapa educativa. Por su parte, la selección de las iniciativas se ha hecho con base en las fuentes documentales y trabajos precedentes consignados en la introducción de este trabajo, a las que se les ha sumado aquellas iniciativas que los autores han considerado oportunas a partir de sus experiencias o bien son demanda reiterada del alumnado.

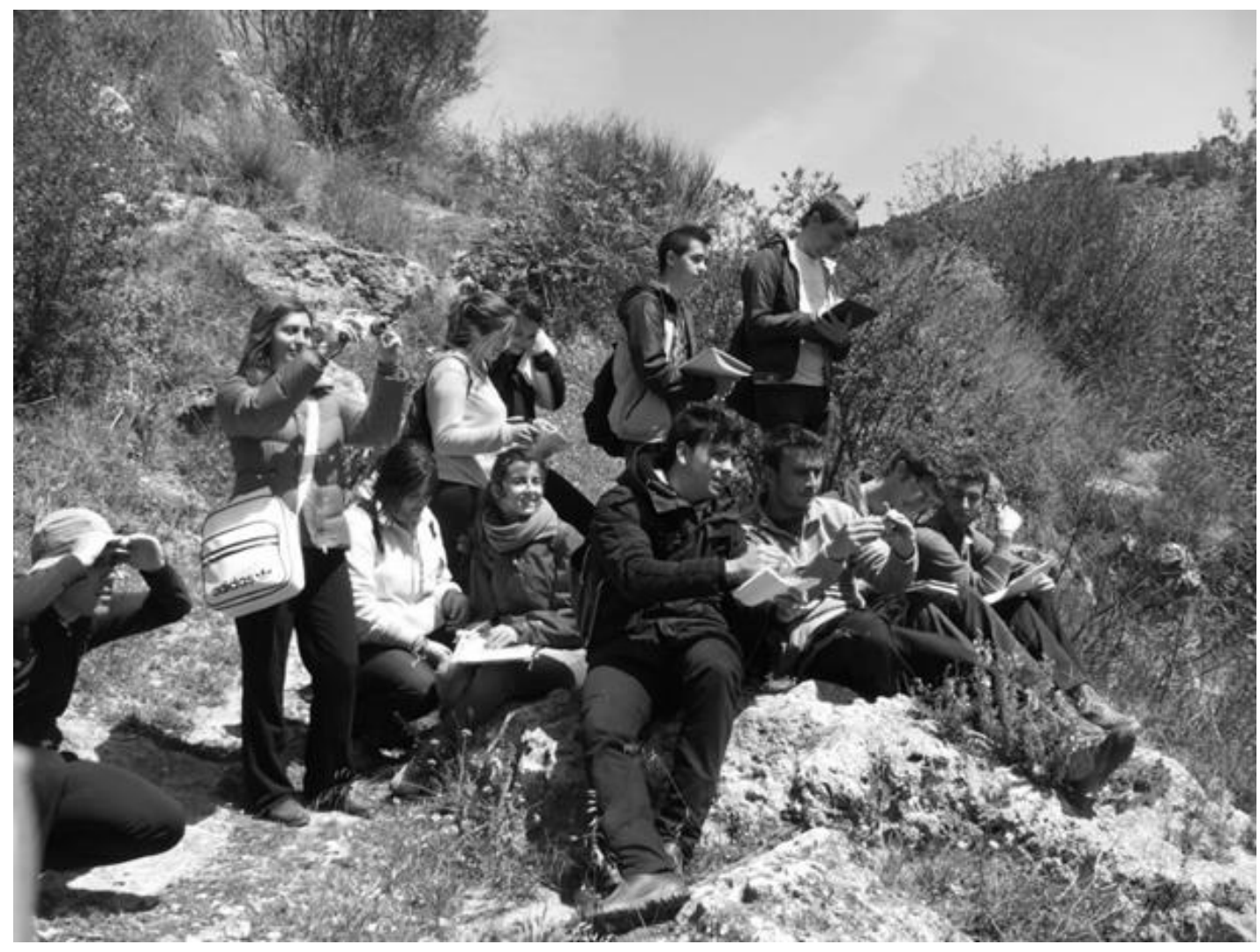

Figura 1: Alumnos de grado (Geografía y Gestión del Territorio de la Universidad de Granada) cumplimentando la Guía de Trabajo en Cónchar (Granada) durante la salida de campo del curso 2017-2018. 


\section{Resultados}

Consecuencia de todo ello es la tabla base sobre la que se consignan las distintas relaciones encontradas (Tabla 1) y, sobre todo, tras la misma, la justificación de los pronunciamientos favorables o desfavorables atribuidos (apuntados como una predisposición positiva, negativa o indiferente ante cada iniciativa); interpretaciones que, aun careciendo de un refrendo estadístico más exhaustivo, objeto éste que requiere un estudio más profundo, se consideran suficientes para iniciar o lanzar la cuestión, e ir adoptando ya ciertas medidas básicas en la confección de los itinerarios didácticos de las salidas de campo en Geografía y en ordenación del territorio.

La tabla que a continuación se presenta trata de mostrar los principales resultados obtenidos en el trabajo de investigación realizado. En ella se valora cada una de las iniciativas planteadas en la confección de los itinerarios didácticos de las salidas de campo en Geografía por cada etapa educativa. Una valoración positiva significa que el colectivo del pertinente nivel educativo interpreta favorablemente la iniciativa y viceversa en el caso de valoración negativa. Cuando no se marca signo es porque la iniciativa resulta indiferente o no se tiene constancia de apreciaciones a favor o en contra. Tras la tabla se fundamentan y comentan los principales resultados obtenidos.

Tabla 1: Iniciativas planteadas en la confección de los itinerarios didácticos de las salidas de campo en Geografía y en urbanismo/ordenación del territorio por nivel educativo.

\begin{tabular}{|c|c|c|c|c|c|c|}
\hline INICIATIVAS & \multicolumn{6}{|c|}{ NIVEL EDUCATIVO } \\
\hline & $\begin{array}{l}\frac{\pi}{\frac{\pi}{\pi}} \\
\stackrel{\frac{\pi}{2}}{\frac{2}{2}} \\
.\end{array}$ & 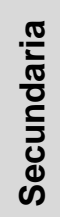 & $\begin{array}{l}\frac{0}{0} \\
\frac{\pi}{0}\end{array}$ & 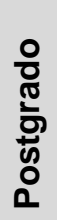 & $\begin{array}{l}\text { E் } \\
\frac{0}{0} \\
\frac{\pi}{2} \\
\frac{\pi}{\alpha}\end{array}$ & 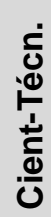 \\
\hline Itinerario consensuado & & + & + & + & & - \\
\hline Itinerario co-diseñado & & & + & + & + & - \\
\hline Itinerario co-elaborado & & + & & + & + & - \\
\hline Itinerario atractivo & + & + & + & + & + & + \\
\hline Aportación de material previo & + & & & + & + & + \\
\hline Seguridad del itinerario & - & - & & & - & \\
\hline Adaptación a las posibilidades físicas & + & & & & - & \\
\hline Contenidos adaptados al tiempo disponible o previsto & - & & & & - & - \\
\hline Contenidos adaptados a la distancia & & & & & - & \\
\hline Contenidos adaptados a las circunstancias & - & & & & - & - \\
\hline Explicaciones localizadas & + & + & + & & + & \\
\hline Explicaciones deslocalizadas & - & - & & + & & + \\
\hline Previsión de recesos no lectivos & - & - & & & + & + \\
\hline Participación durante el itinerario & + & + & + & + & + & + \\
\hline Trabajos in situ & - & - & + & + & - & + \\
\hline Trabajos de recuerdo (memorias, resúmenes...) & + & + & & & + & - \\
\hline Evaluación de la salida de campo & & + & + & + & - & - \\
\hline
\end{tabular}

- Itinerario consensuado: Determinar a dónde y por qué se va a ir a un lugar concreto es parte fundamental en la confección de un itinerario didáctico. Que exista un acuerdo tácito al respecto, o al menos mínimo, favorece la implicación del alumnado. Esta implicación es muy positiva en las etapas educativas más bajas -a excepción de Primaria donde aún no se tiene plenas capacidades para ello y a lo sumo ésta corresponde a los padres a través del Consejo Escolar-, pero tiene menos beneplácito cuando se trata de convenciones científico- 
técnicas, en las que la escasa disponibilidad de tiempo de los asistentes hace que éstos prefieran delegar en los organizadores la gestión del itinerario didáctico; así se desprende de diversas experiencias al respecto, una de las más recientes la ocurrida con motivo del II Coloquio Internacional de Geografía Rural, cuando al solicitar la colaboración para trazar los Itinerarios geográficos en la Granada rural (Cejudo, Navarro y Arias, 2018) apenas se recibieron aportaciones.

- Itinerario co-diseñado: Además de consensuarse, un itinerario didáctico ha de diseñarse en su recorrido. Esto requiere ya un mínimo de información y documentación, de ahí que sea especialmente productivo en los niveles formativos universitarios, incluido el del Aula Permanente, por la especial motivación de los discentes -aunque por las características tan heterogéneas del alumnado resulta complicada su aplicación-, mientras que al igual que sucede en el caso anterior, a nivel científico-técnico es menos propicio. En el caso particular de la educación Primaria esta iniciativa se suele emplear para implicar a los padres en la elección de los puntos a visitar, haciéndolos coincidir, en la medida de lo posible, con lugares en los que los padres de los alumnos desarrollan algún tipo de actividad afín (laboral, recreativa, hobby, etc.)

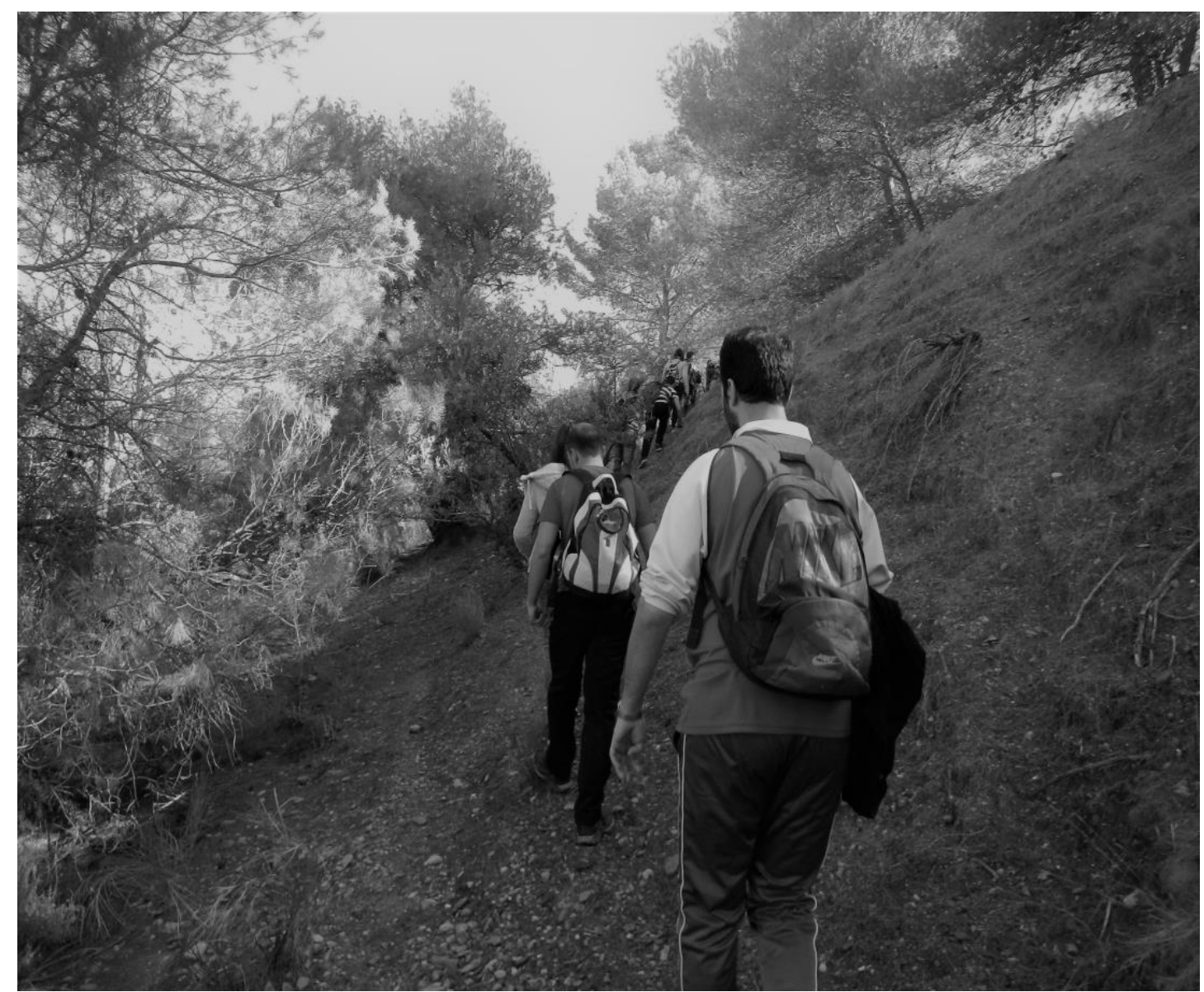

Figura 2: Alumnos de postgrado (Máster en Análisis y Gestión del Territorio de la Universidad de Granada) recorriendo piedemontes y medias laderas en Sierra Nevada (Granada) durante la salida de campo del curso académico 2017-2018. 
- Itinerario co-elaborado: Una vez concretado el destino y diseñada la visita, ha de dotarse de contenido formativo, siendo esto lo que se ha denominado "elaboración del itinerario didáctico". Como resulta obvio, es imprescindible un mínimo de información y documentación, incluso de estudio e interpretación, del itinerario en cuestión, para poder así complementarlo adecuadamente -en realidad, la mayoría de las salidas de campo, tanto en Geografía como en las demás disciplinas que las incluyen en sus programas formativos, esta labor se ha realizado desde siempre por parte de los docentes encargados de las mismas-. Por ello, en las etapas más bajas difícilmente podrá ser atendido como debiera, ya que el alumnado aún está en fase de formación propedéutica, mientras que en los niveles más avanzados resulta más factible, pues cuanto menos los alumnos de postgrado ya se están orientando a la investigación en su Trabajo Fin de Máster o en su Tesis Doctoral. No obstante, es recomendable en Enseñanza Secundaria, no tanto por la calidad del resultado, sino por el hecho de hacerlos personalmente co-responsables de las decisiones que adopten en su formación.

- Itinerario atractivo: La circunstancia de que el recorrido, y lo que se va a vivir durante el mismo, sea capaz de seducir al alumnado es fundamental en todas las etapas (Fernández, 2017: p.106). No obstante, hay que precisar que este aprecio tiene distintas consideraciones según se trate de un nivel u otro. Así, en la enseñanza preuniversitaria, tanto Primaria como Secundaria, prevalecen las vivencias que se van a derivar del itinerario; mientras que en la permanente y en la científico-técnica lo primordial es que tenga capacidad de descubrir nuevos aspectos y puntos de vista sobre una cuestión o sobre un territorio dado. Además, estaría el hecho nada desdeñable de que, normalmente, en la enseñanza pre y universitaria estas salidas suelen ser obligatorias, mientras que en las restantes no lo son, circunstancia que dice mucho sobre la motivación y la entrega al aprendizaje que pudieran tener, en general, unos y otros.

- Aportación de material previo: Supuesto que es muy importante documentarse antes de afrontar el itinerario didáctico, previamente se han de recabar buena parte de aquella información que puedan facilitar la compresión de los elementos, hechos y procesos a analizar in situ. Tal documentación será esencialmente bibliográfica (libros, artículos, informes, etc.), gráfica (mapas topográficos, mapas temáticos, ortofotografías, fotografías, dibujos, diagramas, etc.), estadística, planificadora y legislativa; y cristalizará en una "Guía de trabajo" (Pérez y Rodríguez, 2006, p.232) a partir de la que se iniciará el itinerario. Según se deriva de la experiencia adquirida, esta documentación es más productiva en las etapas superiores, pues se es más propicio a documentarse previamente a la salida de campo, y a sacar el máximo partido de la misma en términos de aprendizaje, que, en los niveles inferiores, en los que se considera como cuestión subsidiaria, toda vez que predomina, entre los discentes, el interés por las experiencias puramente sensoriales y de relaciones interpersonales. Además, su preparación requiere de una selección previa que resulte debidamente adaptada a la etapa a la que se dirige, pues hay que tener en cuenta que, en algunos casos, como el de la Educación Primaria, habrá incluso que primar el aprendizaje de qué es un mapa y cómo se interpreta, al del contenido mismo de éste, por poner sólo un ejemplo. Circunstancia que debe ser 
aprovechada para ir familiarizando al alumno con aquellos materiales más habitualmente utilizados en Geografía.

- Seguridad del itinerario: La legislación sobre seguridad en los itinerarios está considerada en el corpus jurídico andaluz ${ }^{1}$ y de la propia Universidad de Granada $^{2}$, si bien no está muy desarrollada, limitándose a garantizar que estas salidas se realicen en un entorno seguro y en relación con las actividades que se organizan. Habitualmente suelen dar más problemas las salidas de campo de los niveles educativos dirigidos a los alumnos más jóvenes y mayores, porque suelen ser más incautos (Wess, 1990) o se encuentran más limitados, respectivamente. De hecho, la seguridad es uno de los principales problemas que se encuentran los alumnos del Aula Permanente de Formación Abierta (Maroto, Cejudo, Navarro, Cañete y Sánchez, 2015), quienes cuando cumplen más de 65 años deben aportar un Certificado Médico Oficial donde se especifique claramente que el interesado es apto para realizar actividades físicas; y en la educación Primaria, que exige la autorización previa y por escrito de los padres. En cualquier caso, resulta obvio que en toda salida de campo deben contemplarse todas las medidas posibles para minimizar el riesgo de accidentes u otro tipo de problemas.

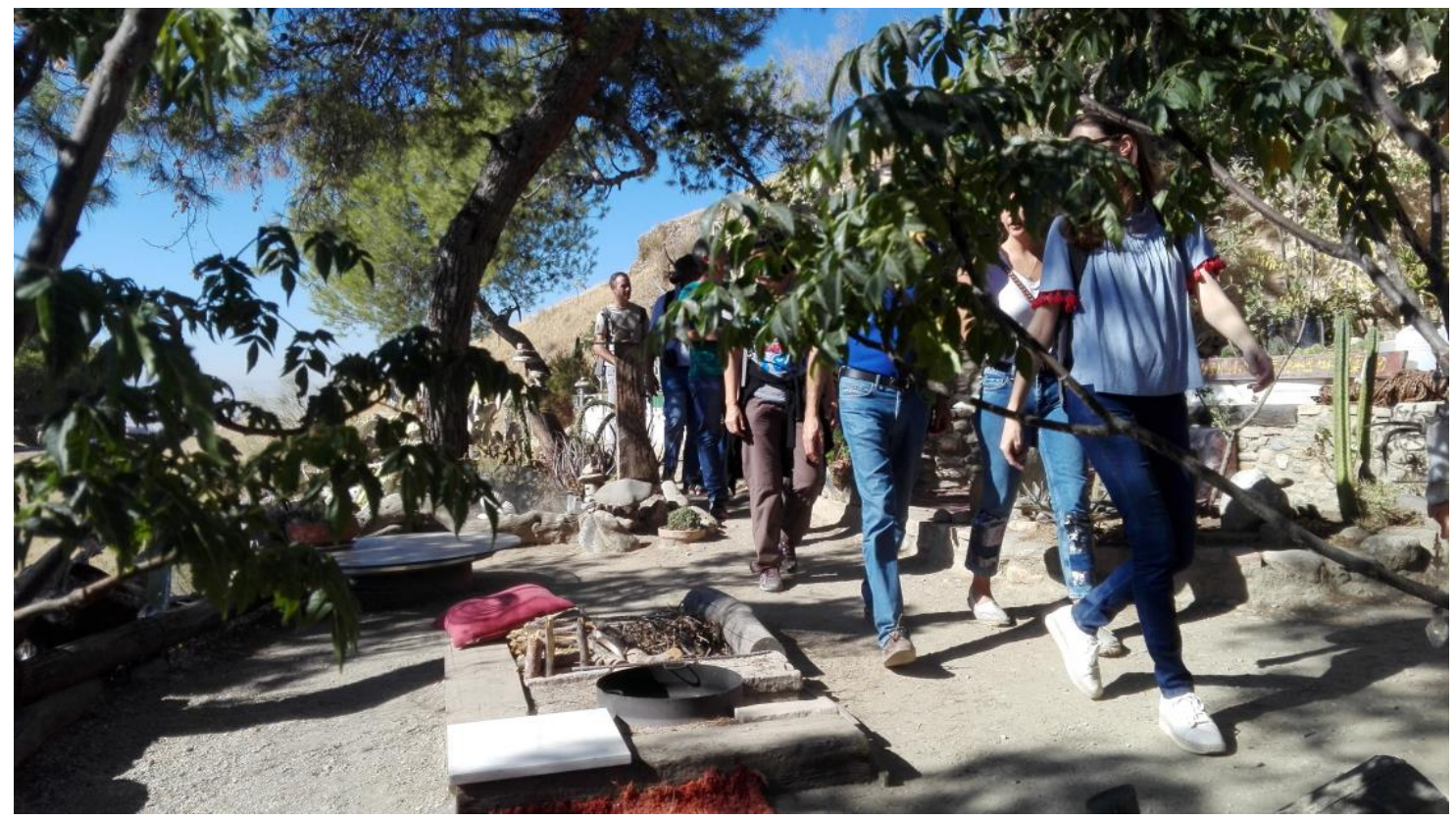

Figura 3: Alumnos de prácticas del Máster en Rehabilitación Arquitectónica y del Máster en Tutela del Patrimonio Histórico-Artístico "El Legado de Al Ándalus" durante una salida de campo por el Sacromonte junto al equipo redactor del Plan Especial de Protección del Albaicín y Sacromonte durante el curso 2017-2018.

\footnotetext{
1 Ley 3/2017, de 2 de mayo, de regulación de los senderos de la Comunidad Autónoma de Andalucía.

${ }^{2}$ Normativa de participación en actividades de montaña. Centro de Actividades Deportivas. Universidad de Granada.
} 
- Adaptación a las posibilidades físicas: Precisamente por las restricciones normativas antes expuestas, todos los alumnos, y en su caso sus tutores, tienen que estar al corriente de la actividad que van a realizar, en particular sobre los requerimientos físicos y técnicos mínimos que ésta exige. En este sentido, por lo general, cuanto más jóvenes son los alumnos, mayores son sus prestaciones físicas, mientras que en los itinerarios planteados para las personas mayores hay que tener muy en cuenta sus limitaciones, siendo un importante hándicap para el desarrollo de ciertos itinerarios, por lo que es preciso adaptarlos, aunque ello implique reducción de posibilidades de observación y aprendizaje sobre la zona visitada. En cualquier caso, hay que reconocer que la práctica de actividad física aparejada a las salidas de campo es una muy recomendable iniciativa biosaludable para prácticamente todos los niveles educativos, previniendo en los niveles iniciales, en coordinación con el médico del Equipo de Orientación Educativa de referencia, problemas tales como la obesidad (Agencia Española de Consumo, Seguridad Alimentaria y Nutrición, 2016), y en las etapas finales otras enfermedades crónicas (Instituto de Mayores y Servicios Sociales, 2014).

- Contenidos adaptados al tiempo disponible o previsto: Atenerse a los tiempos preestablecidos es crucial para el correcto y completo desarrollo de los itinerarios didácticos, siendo interpretada la reducción o dilatación del tiempo fijado como una improvisación de este y, en consecuencia, como una flagrante falta de planificación. Esta carencia suele suscitar más crítica entre quienes tienen la agenda más comprometida, normalmente los alumnos de la enseñanza permanente y científico-técnica, mientras que tiene menos trascendencia entre los que aún están recibiendo una formación reglada, aunque siempre hay tener muy en cuenta el cumplimiento de los horarios en los que los padres dejan y recogen a los alumnos menores. Además, a mayor tiempo ocupado en la salida, menor grado de atención prestada; de ahí la importancia de ajustarse a los tiempos pre-establecidos, sobre todo en Primaria.

- Contenidos adaptados a la distancia recorrida: A semejanza del tiempo, variaciones en la distancia recorrida suelen aparejar una baja valoración por parte del alumnado, ya que impide observar todo lo que estaba previsto o dilata excesivamente la estancia en el medio de transporte empleado, generalmente restándose de las paradas programadas. Esta eventualidad resulta especialmente gravosa para los alumnos mayores, que en algunos casos se quejan del excesivo tiempo empleado en el desplazamiento, de la escasa información recibida directamente del campo y de lo inasumible físicamente que pueden resultar los recorridos excesivamente largos. No obstante, la posibilidad de aprovechar el desplazamiento motorizado (normalmente en autocar) para realizar comentarios, a ser posible amenos, por parte del docente sobre lo que se percibe a lo largo del recorrido -lo que se ha venido a denominar "el paisaje en movimiento"-, atenúa en gran parte la rémora mencionada. Otra opción es la de brindar a los discentes la posibilidad de comentar aspectos concretos del recorrido, si bien suelen ser reticentes en la mayor parte de los niveles educativos. 
- Contenidos adaptados a las circunstancias ambientales: Con frecuencia, los itinerarios didácticos de las salidas de campo se ven alterados por las condiciones ambientales del recorrido o de la jornada. Quizás el caso más paradigmático sea el de las inclemencias meteorológicas, que pueden dar al traste con cualquier tipo de planteamiento. Es por ello que prever, en la medida de lo posible, esta circunstancia, o bien plantear de manera anticipada soluciones alternativas, se recomienda encarecidamente, en especial cuando se trata de docencia en las etapas del Aula Permanente, científico-técnica y Primaria, en el primer caso porque son altamente sensibles a condiciones ambientales duras, en el segundo porque suelen llevar la agenda muy ajustada y no pueden permitirse variaciones sustanciales en ella, y en el tercer caso porque una inadecuada equipación del alumnado puede suscitar la queja colectiva de los padres, que aunque suelen ser previsores, no siempre aciertan con la vestimenta recomendada o, más frecuente aún, se exceden en la misma.

- Explicaciones localizadas: Durante el desarrollo de un itinerario didáctico se pueden ofrecer explicaciones localizadas (cuando se está ante los hechos que las motivan) o deslocalizadas (de contexto y no necesariamente inmediatas ni en el espacio ni en el tiempo). Las explicaciones localizadas son muy agradecidas entre los niveles educativos de pregrado, grado y del Aula Permanente, que es donde por lo general suelen tenerse mayores dificultades para realizar grandes abstracciones sobre las distintas materias territoriales; de ahí que sea más conveniente aplicar aquí las explicaciones de forma sincrónica que diacrónicamente. En cualquier caso, puede afirmarse que las salidas de campo adquieren mayor valor didáctico y mayor justificación cuando ofrecen explicaciones localizadas y bien articuladas discursivamente; esto es, adecuando las explicaciones y las actividades a cada tramo del recorrido y a cada una de las paradas previstas (Sánchez del Árbol, 2009).

- Explicaciones deslocalizadas: Al contrario de lo que sucede con las explicaciones localizadas, las etapas educativas más elevadas -el postgrado y el científico-técnico-, están mejor cualificados para recibir fundamentaciones con un mayor grado de abstracción, de forma que no es imprescindible contar con la proximidad de los hechos que se narran para su adecuada comprensión. De hecho, se ha constatado que las explicaciones deslocalizadas entre los alumnos de postgrado incrementan considerablemente la relación de fenómenos (Garrido, Sánchez y Cuesta, 2017). No obstante, tampoco tiene mucho sentido la aplicación generalizada de las explicaciones deslocalizadas, pues desvalorizaría o restaría interés al itinerario programado en la salida de campo. En todo caso, en etapas educativas iniciales, como la Primaria y la Secundaria, si no se perciben los motivos geográficos que motivan el desplazamiento, es mucho más difícil que éstos sean aprendidos por el alumnado.

- Previsión de recesos no lectivos: Cuando las salidas de campo son muy dilatadas en el tiempo es recomendable prever recesos para el descanso, el avituallamiento o el aseo. Estos han de servir para atender las necesidades biológicas básicas, pero también para producir momentos de desconexión en la docencia que ayuden a recuperar la atención del alumnado una vez reiniciado el 
itinerario. Estas paradas estratégicas serán menos necesarias cuanto más jóvenes sean los alumnos, pudiendo llegar a ser incluso desaconsejables en la Enseñanza Primaria y Secundaria si conlleva la ruptura del ritmo en el proceso enseñanza-aprendizaje (en estos niveles es preferible programar salidas de campo de corta duración). Por el contrario, tales recesos son prácticamente inevitables entre el alumnado mayor, que no sólo tiene más requerimientos fisiológicos, sino que encuentra en estos momentos una gran ocasión para sociabilizarse -escuchar y ser escuchados-, así como para inquirir al profesorado alguna cuestión que, por timidez, posible rubor o por su carácter confidencial, no le haya sido formulada en su momento.

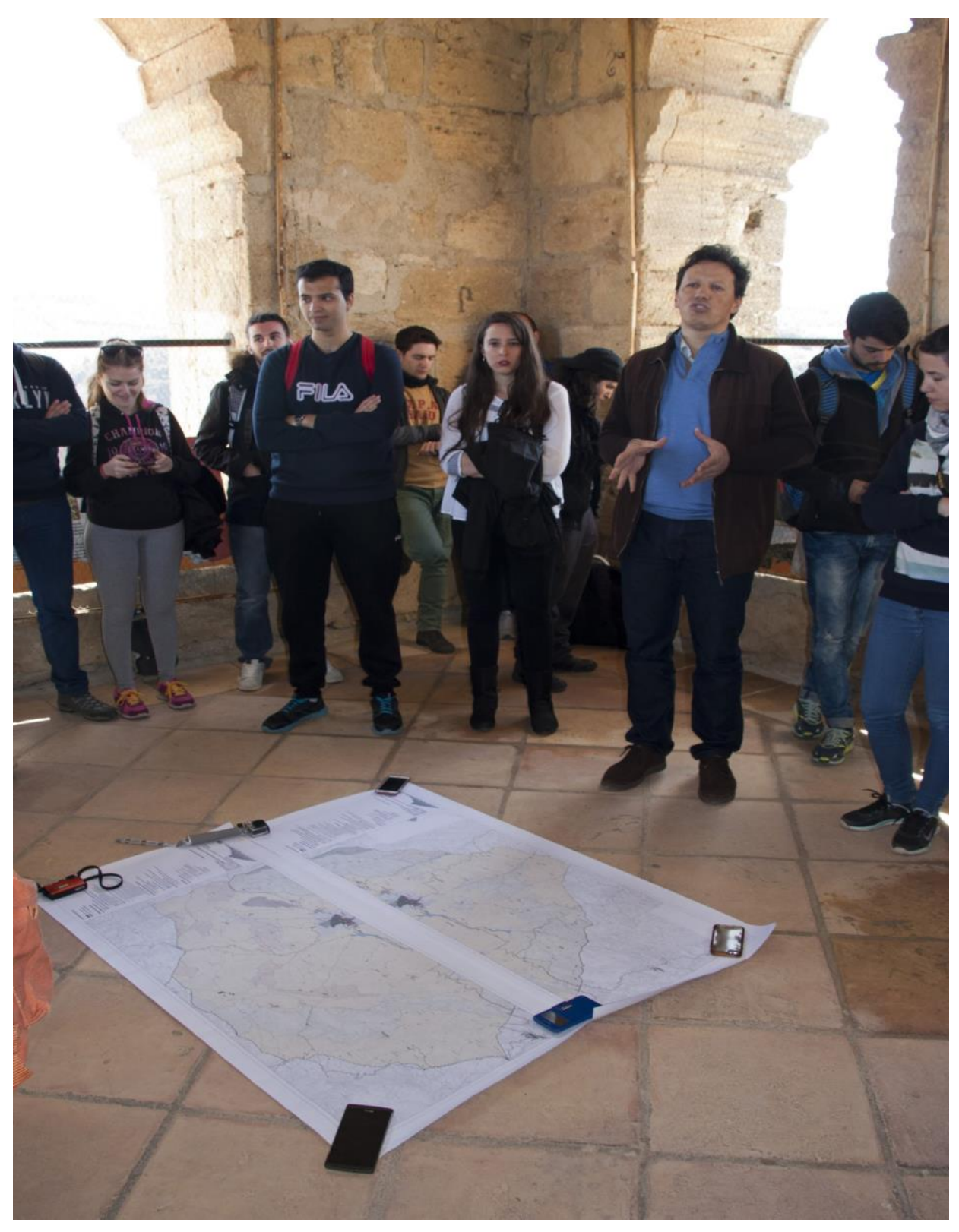

Figura 4: Alumnos de postgrado (Máster en Análisis y Gestión del Territorio de la Universidad de Granada) recibiendo explicaciones deslocalizadas desde el campanario de Montefrío (Granada) durante la salida de campo del curso académico 2016-2017 (Jordi Blay, 2017). 
- Participación durante el itinerario: Una mayor participación individual o colectiva durante el desarrollo efectivo de la salida significará implicación del alumnado, retroalimentará las relaciones alumno-profesor e incluso alumnoalumno, y favorecerá una dinámica fluida de la misma ${ }^{3}$. No quiere decirse con ello que la mayor parte del peso del itinerario didáctico deba recaer sobre el alumnado, pero sí que estas salidas de campo deban ser un tanto "autodirigidas" ${ }^{4}$ por el alumnado -al menos, co-dirigidas, co-participadas o coresponsabilizadas entre docente y discentes ${ }^{5}$-, lo que habrá de suponer un incentivo para el mismo. Así pues, habrá de propiciarla en las enseñanzas básicas, pudiéndose incluso obligar a esa co-participación llegado el momento, y debería contenerse en la de mayores, ya que el excesivo afán participativo y decisional de algunos participantes podría romper el ritmo o la propuesta de contenidos del itinerario. Sin embargo, es precisamente en los niveles universitarios de grado y posgrado donde puede resultar a priori más provechosa la co-participación dado el nivel de madurez intelectual, aunado a la presumible motivación al tratarse de niveles de enseñanza no obligatoria.

- Trabajos in situ: El pedir la realización de trabajos relacionados con el itinerario didáctico in situ puede tener efectos pedagógicos tanto favorables como perjudiciales. Favorables o positivos en tanto que se podría profundizar sobre la temática a partir de los datos aportados por la "presencialidad", que se facilitaría el aprendizaje de los factores que interactúan al detectarse más claramente en la proximidad, que se produciría un mayor acercamiento y reconocimiento a los diferentes actores sociales intervinientes sobre el territorio (Pérez y Rodríguez, 2006, p.233) y que las aportaciones del colectivo podrían servir para contrastar las conclusiones sobre el terreno. En contra o negativos por cuanto que implica consumir mucho tiempo de la salida y, de no organizarse adecuadamente, podría dirigirse hacia el caos, especialmente en los casos de la Enseñanza Primaria y Secundaria. Con estas premisas, el trabajo in situ es más idóneo para los niveles educativos más académicos y científico-técnicos, que resultarían ser los más operativos al respecto, y menos adecuados para los niveles educativos iniciales y destinados a los alumnos mayores.

\footnotetext{
${ }^{3}$ Desde el punto de vista pedagógico, el trabajo de campo no puede orientarse a la simple transmisión de una información elaborada por el profesorado sobre un objeto o espacio geográfico, sino que el alumnado ha de adquirir una actitud proactiva, y así alcanzará una mayor conciencia del paisaje del ámbito de estudio, de su concepción holística y de los problemas conexos; una comprensión básica del paisaje en su complejidad, lo que entraña una responsabilidad crítica y propositiva; y la capacidad para clasificar, identificar y caracterizar los paisajes en función de los factores ecológicos, económicos, sociales, estéticos, perceptuales, etc. (Jerez García, 2007).

4 "En ellas el estudiante es autónomo a la hora de la investigación (son ellos mismos quienes seleccionan el área a estudiar, planifican los objetivos, buscan el material, diseñan el itinerario, etc.). El trabajo puede ser realizado individualmente o en grupos pequeños y el rol del profesor es meramente tutorial. A pesar de que la actuación del profesor se ve minimizada, en ningún momento se debe descartar la importancia que su participación tiene pues el éxito de las actividades de los estudiantes dependerá de una buena asesoría" (Godoy y Sánchez, 2007, p.142).

${ }^{5}$ A tenor -entre otras referencias- de la propuesta elaborada por Sánchez del Árbol, M.A. (2011) en el trabajo titulado: "Definición de una metodología para el fomento e integración de las participación activa del alumnado en el trabajo de campo en el Grado de Geografía y Gestión del Territorio" (dentro de la Acción n²: "Innovación de metodologías docentes para clases teóricas y prácticas"), dentro del Curso sobre Incorporación de metodologías activas al aula universitaria, organizado por el Vicerrectorado para la Garantía de la Calidad, Universidad de Granada, con motivo de la implantación del EEES. En esta aportación se define, entre otros aspectos, la distribución docente-discente de cada una de las etapas de la preparación (pre-salida), ejecución (salida) y resultado (post-salida) del trabajo de campo geográfico.
} 
- Trabajos de recuerdo (memorias, resúmenes, comentarios...): Tras el desarrollo del itinerario didáctico y la realización de su correspondiente salida de campo, en algunos casos es muy recomendable realizar un trabajo en el que se recojan las principales conclusiones o impresiones obtenidas durante el mismo e incluso su presentación y debate ante la comunidad (Pulgarín, 2000, p.5). Éste suele emplearse para asegurar la atención durante la actividad docente, en especial a nivel de Primaria, Secundaria y de Grado, mientras que en postgrado y permanente puede resultar muy útil como autoevaluación del itinerario. No es muy recomendable a nivel científico-técnico, pues se supone que el aprendizaje adquirido se aprovechará particularmente $y$, en consecuencia, exigirlo se considera demeritorio.

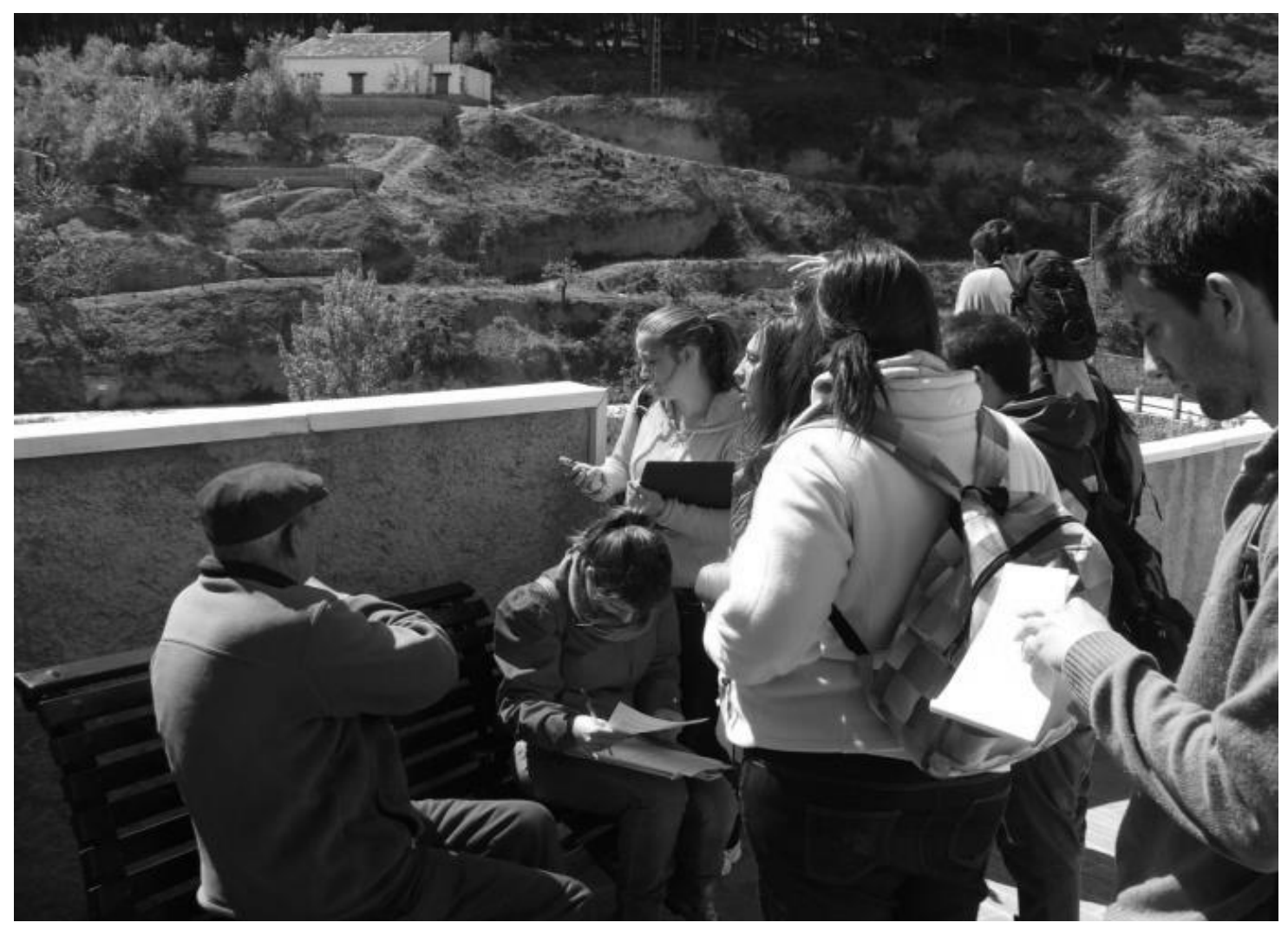

Figura 5: Alumnos de grado (Geografía y Gestión del Territorio de la Universidad de Granada) entrevistando a agentes locales en Albuñuelas (Granada) durante la salida de campo del curso académico 2017-2018.

- Evaluación de la salida: Como se ha expuesto en el apartado anterior, los trabajos de recuerdo o un documento de evaluación expreso resultaría muy conveniente para mejorar el itinerario didáctico en cuestión, así como los sucesivos. Sin embargo, éstos no tienen buena acogida entre los alumnos del Aula Permanente y menos aún en el colectivo científico-técnico, por cuanto que muy posiblemente ya no volverán a repetir el itinerario, además de percibirse como tarea onerosa por parte de la mayoría del alumnado; y, en el segundo caso particularmente porque, en general, no suelen querer pronunciarse ante unos circunstanciales compañeros de viaje -con los que, en todo caso, prefieren 
mantener una relación de cortesía-, pues es más probable que prevalezca la competencia sobre la colaboración entre ellos. No obstante, tal evaluación resulta muy adecuada para el resto de etapas educativas, ya que favorece su implicación en el buen discurrir del ciclo formativo que les reste por completar, porque refrenda las decisiones del profesorado encargado de diseñar y elaborar los futuros itinerarios didácticos (García-Martín, Villar, Fraile, Sánchez y Márquez, 2018, p.122) y porque " con ella se produce un feedback donde se evalúa al alumnado y, a su vez, el alumnado evalúa el proyecto, facilitándose así la mejora continua de la actividad" (Mohamed, Pérez y Montero, 2017, p.194).

\section{Discusión}

Como ha podido comprobarse, en la Enseñanza Primaria son muy positivas las iniciativas que están relacionadas con hacer cuanto más sugerente posible los itinerarios, tanto en su diseño como en su desarrollo y posterior evaluación; para ello siempre es muy aconsejable hacer reiteradas referencias al mismo en cada momento, por ejemplo creando expectativas del tipo "ya veréis que interesante" en clases previas, recalcando expresiones del tipo "no os dije que os iba a gustar" durante el desarrollo de la actividad, o trayendo a colación referencias del tipo "tal y como vimos cuando fuimos alli" una vez realizada la salida de campo. El resto de iniciativas deben orientarse a estas pretensiones, dotándolas de la mayor interactividad entre alumnos, docentes y padres, en pro de la tranquilidad de todas las partes frente a la incertidumbre que se les vislumbra ante una práctica que no deja de ser novedosa para todas las partes en estas etapas educativas (Wass, 1990).

En la Enseñanza Secundaria se debería dar continuidad a lo expuesto para la Primaria, si bien haciendo que la interrelación entre alumnos sea lo más creativa posible y que la interactuación con el territorio sea lo más implicada posible. Para ello es importante que el alumnado vaya tomando decisiones en cuanto al itinerario, de manera que cobre responsabilidades, uno de los retos para las poblaciones adolescentes actuales (Echegaray, 2013). Esto se puede lograr haciendo que consensuen y co-diseñen el itinerario, participen en la elaboración del material previo a utilizar, se les incite a intervenir durante el itinerario y expongan sus respectivos trabajos de recuerdo y evaluaciones ante el resto de compañeros, de manera que puedan precisarse y debatirse las discrepancias surgidas. Ítem aparte, esta será una gran oportunidad para suscitar y potenciar entre el alumnado cambios de comportamiento como el interés y curiosidad por identificar, relacionar y comprender los elementos constitutivos del territorio, la sensibilidad/rechazo ante las desigualdades sociales y la marginación, el compromiso con la conservación ambiental, cultural y patrimonial, el rigor en la recolección de la información, el espíritu crítico, etc. (Pérez y Rodríguez, 2006, p.232).

Respecto a la enseñanza de Grado quizás la parte en la que habría que hacer más hincapié es en la preparativa (consenso, co-diseño y co-elaboración del itinerario) y la posterior (trabajo de recuerdo y evaluación de la salida). En esta etapa es tan importante el aprendizaje que se logra con la realización del itinerario, fundamentalmente aspectos territoriales, como el hecho de aprender la sistemática empleada para su desarrollo, pues no hay que perder de vista que los futuros graduados tendrán que implementar itinerarios tanto en su vida profesional, ya sea en el sector educativo como en el técnico, como en su vida personal, por ejemplo a la hora de tramar un viaje. A ello coadyuvará que hayan pasado por la experiencia de haber diseñado previamente un itinerario, así como el hecho de que lo hayan pasado por el tamiz de la auto-crítica, siempre con afán de retroalimentarlo y mejorarlo constantemente. 
En Postgrado también interesan los trabajos previos a la confección del itinerario, pero donde más se aprovechan es durante el desarrollo de este, ya sea a través de la participación activa en él, ya sea mediante la realización de trabajos in situ. Las vivencias y el aprendizaje en tiempo real son muy importantes para el alumnado de postgrado porque ayuda a desvelarle aspectos del territorio que sólo aparecen en la relación a escala uno-uno, y sobre todo porque propicia el enfoque de los conocimientos adquiridos hacia la temática de su Trabajo Fin de Grado, Trabajo Fin de Máster, Proyecto de Investigación o Tesis Doctoral. En definitiva, se trata más que de una salida de campo, de un campo de trabajo, de experimentación, con el que refutar las hipótesis que se plantea en sus futuras investigaciones.

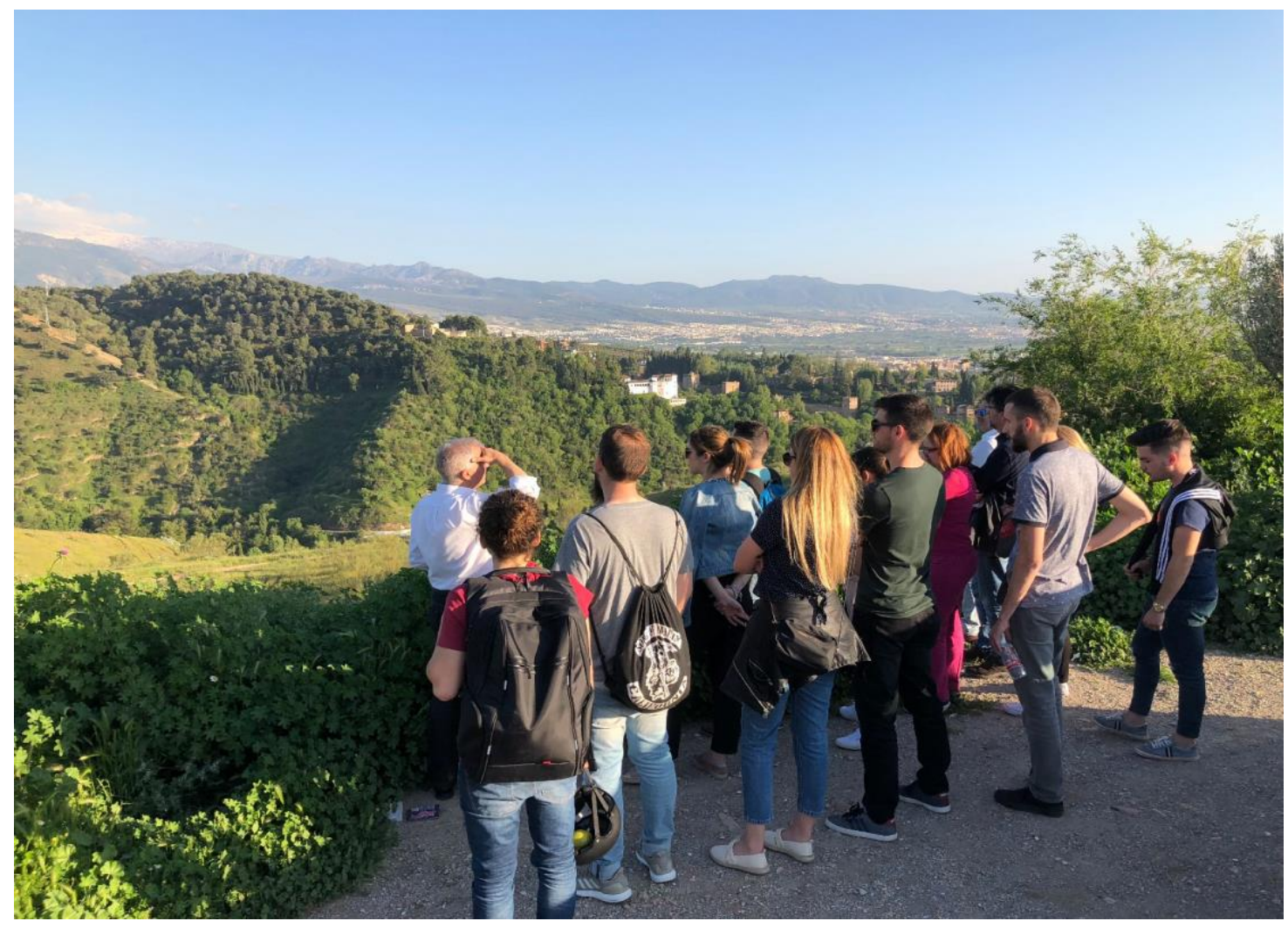

Figura 6: Alumnos del Grado de Edificación (ETSIE/UGR) de la asignatura de Proyecto de Fin de Grado (Línea de Patrimonio y Medio Ambiente) durante la salida de campo del curso 20172018 (Autor Prof. Lafuente Bolívar FJ).

Los estudiantes del Aula Permanente de Formación Abierta, por sus peculiaridades (Sánchez y Garrido, 2019) son más dados a tratar con detalle las cuestiones que rodean al itinerario que a su propio contenido, sin querer descartar con ello el provecho que obtienen de sus enseñanzas. Así, supuesto que es una actividad más ilustrada que formativa dado que las interacciones entre alumno-profesor y alumno-alumno son quizás más valoradas que la transmisión de conocimientos en sí misma (Garrido y Sánchez, 2019), preocupa sobre todo las condiciones en las que se va a realizar la salida y, curiosamente, los materiales que van a guardar de ésta (aportación de material previo y trabajos de recuerdo). Cuestión aparte es que las salidas de campo son un momento de gran sociabilización; de ahí que conferirles un comedido protagonismo suele tener muy buena acogida y es bastante provechoso, sobre todo cuando pueden aportar conocimiento del territorio donde se desarrolla la salida o experiencias similares. 
Por último, las salidas de campo al nivel científico-técnico suelen ser aprovechadas, sobre todo, durante su realización. Hay que entender que la motivación que lleva a realizarla es el interés del asistente por adquirir un determinado aprendizaje sobre el territorio en cuestión y, a partir de ahí, el poder entablar un intercambio fluido de conocimientos con el resto de participantes y los propios organizadores. En consecuencia, tanto las explicaciones localizadas como las deslocalizadas, así como el tener oportunidad de intervenir en las mismas es parte determinante de la formación a este nivel, resultando todo lo demás más accesorio.

\section{Conclusiones}

Lejos de resultar una propuesta pedagógico-didáctica establecida, acabada, las salidas de campo están en permanente construcción (Moreno, Rodríguez y Sánchez, 2011, p.18). Muy atrás quedó aquel primer reconocimiento del valor de las salidas de campo en la vanguardista Institución Libre de Enseñanza, donde la excursión se erigía como un método pedagógico regenerador, de carácter eminentemente inductivo y con el que se sustituía lo abstracto por lo real y concreto. Hoy la propedéutica de las salidas de campo en Geografía y Ordenación del Territorio obliga a una planificación muy precisa de los itinerarios didácticos a seguir habida cuenta de las limitaciones de tiempo y presupuestarias existentes. Es por ello que esta planificación debe hacerse teniendo muy en cuenta el tipo de alumnado al que se dirige, ya que cada nivel educativo requiere de un diseño y una gestión de los itinerarios didácticos específicos, en el que se atienda a sus particularidades, sus motivaciones y sus posibilidades, que en algunos casos difieren radicalmente supuesto que, para unos, formará parte de su formación y futura proyección laboral, mientras que para otros, es complemento de sus dedicaciones o aficiones, o simple colofón a una vida de amplias inquietudes intelectuales, vivenciales y sociales. En suma, desde la experiencia de la docencia en seis etapas educativas se llega al convencimiento de que, para estimular la inteligencia emocional del alumnado que cursa materias en las que el contacto con el territorio es fundamental, hay que proceder con la previsión de unos itinerarios didácticos lo más adaptados posible a tales etapas. Esta afirmación, que puede resultar obvia, se ha tratado de refrendar pormenorizando uno a uno los muy diversos aspectos a tener en cuenta en las salidas de campo por cada una de las etapas de enseñanza-aprendizaje, poniéndose de manifiesto convergencias y divergencias según tales etapas y, lo más importante a nuestro juicio, la identificación y sistematización de las iniciativas que favorecen un mayor grado de acogida o satisfacción por parte de cada grupo discente. Todo ello con el ánimo de lograr que, como decía Joaquín Costa Martínez, "uno de los resultados más positivos de las excursiones practicadas por los alumnos... era el de acostumbrarlos a objetivar su pensamiento, a expresar por escrito sus ideas, a ordenar y sistematizar sus recuerdos, a reflexionar sobre ellos y a crearse un estilo propio, que sea viva expresión de su individualidad".

\section{Referencias}

Agencia Española de Consumo, Seguridad Alimentaria y Nutrición, 2016. Estudio ALADINO 2015: Estudio de vigilancia del crecimiento, alimentación, actividad física, desarrollo infantil y obesidad en España 2015, Madrid, Ministerio de Sanidad, Servicios Sociales e Igualdad.
Cejudo García, E., Navarro Valverde, FA. y Arias García, J. (editores), 2018. Itinerarios geográficos en la Granada rural, Granada, Editorial Alhulia.

Costa Martínez, J., 1916. Maestro, escuela y patria: (notas pedagógicas). Madrid, Biblioteca Costa. 
Dirección de Evaluación y Acreditación, Agencia Andaluza del Conocimiento, 2016. Informe global de acreditación de títulos (Grado en Geografía y Gestión del Territorio).

Echegaray Aldamiretxebarria, K., 2013. Salidas de campo como recurso en la enseñanza de ciencias de Bachillerato. Una propuesta para Ciencias de la Tierra y Medioambientales del $2^{\circ}$ curso. Trabajo Fin de Máster.

Fernández Portela, J., 2017. La salida de campo como recurso didáctico para conocer el espacio geográfico: el caso de la ciudad de Valladolid y de Soria. Didáctica Geográfica №18, 91-109.

García Ballesteros, A., 1986. Teoría y Práctica de la Geografía. Madrid, Alhambra Universidad.

García Martín, M., Villar Lama, A., Fraile Jurado, P., Sánchez Carnero, N. y Márques Pérez, J., 2018. Se hace Geografía al andar: la salida de campo itinerante y senderista. Didáctica Geográfica, ํำ19, 103-105.

García Ruiz, A. L. (2013): "El proceso de desarrollo de los Itinerarios Geográficos", en Didáctica Geográfica (2), pp. 3-9.

Garrido Clavero, J., Sánchez del Árbol, MA. y Cuesta Guerrero, JM., 2017. "El urbanismo en la comarca del Poniente Granadino" en Cejudo García, E. y Navarro Valverde, F.A., coordinadores. Itinerarios geográficos de posgrado. Cuadernos de campo del Máster en Análisis y Gestión del Territorio. Granada, Ed. Universidad de Granada.

Garrido Clavero, J. y Sánchez del Árbol, MA., 2019. "Intercambio de conocimientos entre profesores y alumnos del Aula Permanente de Formación Abierta: Transmisiones de ida y vuelta" en Libro de Actas de las Jornadas Conmemorativas del XXV Aniversario del APFA de la Universidad de Granada. Granada, Ed. Universidad de Granada.

Godoy, I. y Sánchez, A., 2007. "El trabajo de campo en la enseñanza de la Geografía". Sapiens. Revista Universitaria de Investigación, 2, 137-146.

Instituto de Mayores y Servicios Sociales, 2014. Las personas mayores en España. Madrid, Ministerio de Sanidad, Servicios Sociales e Igualdad.

Jerez García, O., 2007. "Paisaje y Geografía Física. De la investigación científica a la transposición didáctica" en: M.J. Marrón Gaite; J. Salom Carrasco; X.M. Souto González, eds. Las competencias geográficas para la educación ciudadana. Grupo de Didáctica de la Asociación de Geógrafos Españoles, pp. 101-115

Moreno Lache, N., Rodríguez Pizzinato, L. y Sánchez Ardila J., 2011. La salida de campo... se hace escuela al andar. Grupo Interinstitucional de Investigación Geopaideia.

Maroto Martos, JC., Cejudo García, E., Navarro Valverde, F., Cañete Pérez, JA. y Sánchez del Árbol, MA., 2015. "El reto de enseñar Geografía del
Turismo a los alumnos universitarios senior", en De la Riva, J., Ibarra, P., Montorio, R. y Rodrigues, M., editores. Análisis espacial y representación geográfica: innovación y aplicación 1445-1454. Zaragoza, Universidad de Zaragoza-A.G.E.

Mohamed-Mimón, M., Pérez Castro, MA. y Montero Alonso, MA., 2017. Salidas pedagógicas como metodología de refuerzo en la Enseñanza Secundaria. ReiDoCrea, 6, 194-210.

OCDE, 2016. PISA 2015 Resultados Clave. http://www.oecd.org/pisa/pisa-2015-results-infocus-ESP.pdf

Pérez de Sánchez, A.G. y Rodríguez Pizzinato, L.A., 2006. La salida de campo: una manera de enseñar y aprender geografía. Geoenseñanza 11(2). 229234.

Pulgarín Silva R., 2000. La excursión escolar como estrategia didáctica en la enseñanza de la geografía. La Gaceta Didáctica (2).

Sala Sanjaume, M. y Batalla Villanueva, R., 1996. Teoría y métodos en Geografía Física. Madrid, Editorial Síntesis. Colección Espacios y Sociedades.

Sousa Fernandez S., García Monteagudo, D. y Souto González XM., 2016. Educación geográfica y las salidas de campo como estrategia didáctica: un estudio comparativo desde el Geoforo Iberoamericano. Biblio 3W, Revista Bibliográfica de Geografía y Ciencias Sociales, XXI(1), 155.

Sánchez del Árbol, MA., 2009. "Del alto Monachil al bajo Guadalfeo: recorrido geográfico", en J. Gómez Zotano y F. Ortega Alba, eds. El Sector Central de las Béticas: una visión desde la Geografía Física. Editorial Universidad de Granada. Pp. 193-199.

Sánchez del Árbol, MA. y Garrido Clavero, J., 2019. "La Geografía, o el enfoque geográfico, en los programas del Aula Permanente de Formación Abierta" en Libro de Actas de las Jornadas Conmemorativas del XXV Aniversario del APFA de la Universidad de Granada. Granada, Ed. Universidad de Granada.

Wass, S. 1990. Salidas escolares y trabajo de campo en la educación primaria. Ministerio de Educación y ciencia - Ediciones Morata.

Zusman, P., 2011. "La tradición del trabajo de campo en Geografía", Geograficando, 7, 15-32. 